\title{
Une question de qualité!
}

\section{Valérie Rothhardt}

Avocate, cheffe du Bureau d'expertises extrajudiciaires de la FMH

Le fait de disposer d'une assurance de responsabilité civile professionnelle (ci-après "assurance $\mathrm{RC}$ ») adaptée à votre activité est un devoir professionnel. Mais quels sont les aspects dont vous devez tenir compte lors de la conclusion d'une telle assurance? En plus du critère financier, qui joue un grand rôle, la qualité des prestations offertes est un élément central. Sous cet angle, la prise en charge des honoraires de/des expert/s dans le cadre d'une procédure devant le Bureau d'expertises extrajudiciaires de la FMH est indispensable.

\section{Un devoir professionnel}

En tant que médecin indépendant, vous avez l'obligation légale et déontologique d'avoir une assurance RC suffisante pour couvrir les risques liés à votre activité. Cette obligation figure aux art. 40 let. $\mathrm{h}$ de la loi sur les professions médicales (LPMéd) ${ }^{1}$ et 35 du Code de déontologie de la FMH $(\mathrm{CoD})^{2}$. La loi ne définit pas la notion de «couverture adaptée». En effet, celle-ci dépend des besoins particuliers du médecin concerné, l'activité d'un gynécologue-obstétricien ou d'un anesthésiste n'engendrant pas les mêmes risques en matière de responsabilité civile que celle d'un généraliste ou d'un psychiatre.
Mais quels sont les points auxquels vous devez prêter attention lors du choix d'une telle assurance? Comment s'y retrouver parmi une multitude d'offres?

\section{La loi ne définit pas la notion de} «couverture adaptée».

L'aspect financier joue bien évidemment un grand rôle. D'un côté, la couverture doit être assez élevée pour couvrir d'éventuelles prétentions en réparation du dommage subi. Les FMH Services recommandent aux médecins exerçant à titre indépendant un montant assuré d'au minimum 10 millions de francs, ceci indépendamment de leur spécialité ${ }^{3}$. De l'autre côté, il s'agit d'obtenir la prime la moins élevée possible, sachant qu'il y a de grandes différences entre les assurances.

\section{La qualité au premier plan}

Malgré l'importance du critère financier, l'aspect qualitatif ne doit pas passer à la trappe. Selon la FMH, l'une des prestations devant être couverte par votre assurance $\mathrm{RC}$ est la prise en charge des honoraires de/s expert/s
1 Art. 40 let. h LPMéd: Les personnes exerçant une profession médicale universitaire à titre indépendant doivent observer les devoirs professionnels suivants: [...] h. conclure une assurance responsabilite civile professionnelle offrant une couverture adaptée à la nature et à l'étendue des risques liés à leur activité ou fourni des sûretés équivalentes. 2 Art. 35 CoD: Le médecin contracte une assurance responsabilité civile professionnelle suffisante.

3 Cf. l'article de Max Giger et Reinhard Kunz, «Les polices et les conventions sont-elles adaptées aux exigences actuelles?», in Bulletin des médecins suisses 2011;92(20):741.

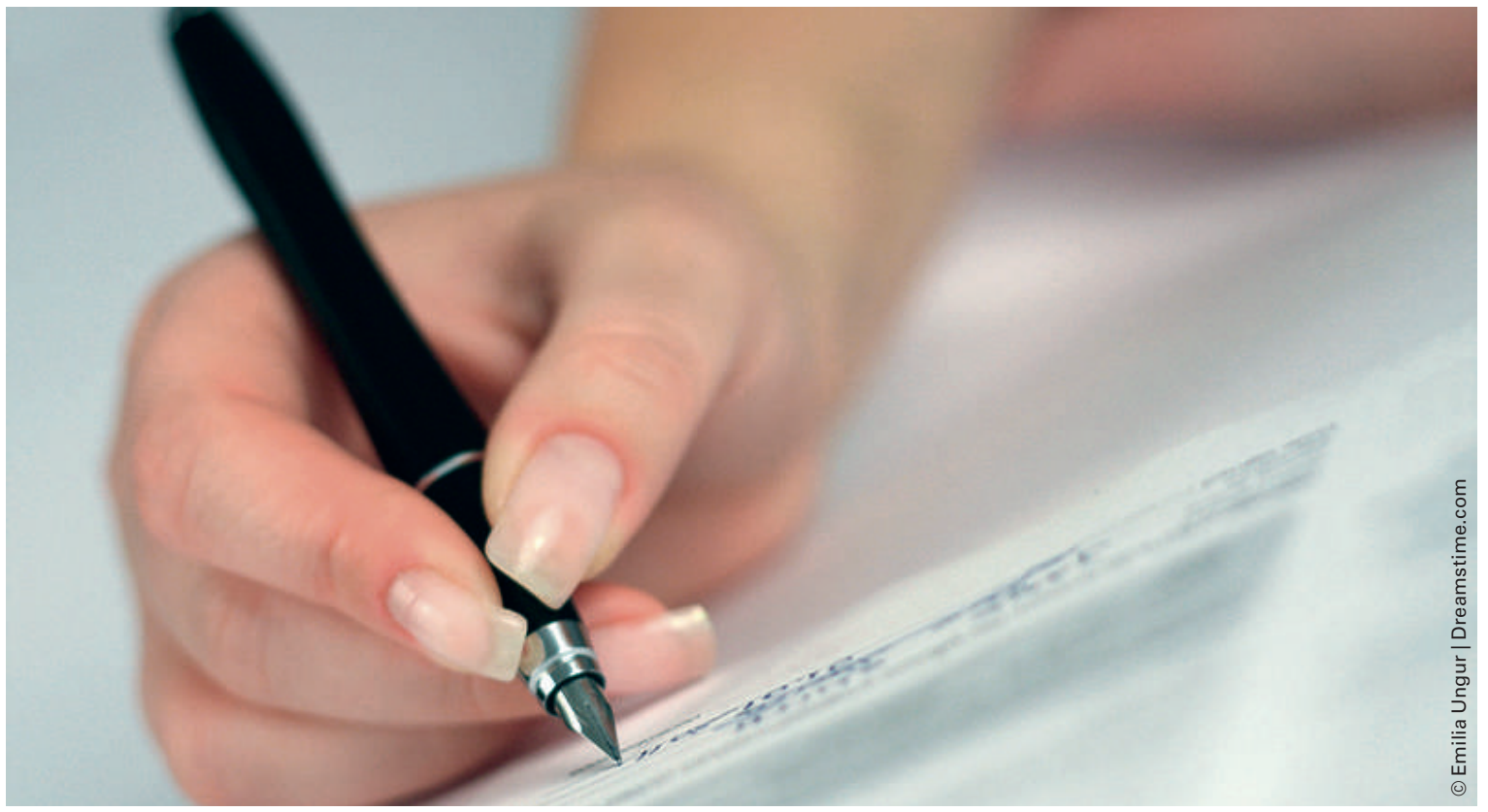

Quels sont les aspects dont vous devez tenir compte lors de la conclusion d'une telle assurance de responsabilité civile professionnelle? 
dans le cadre de procédures devant le Bureau d'expertises extrajudiciaires de la FMH.

Cette prestation doit faire partie intégrante de la qualité offerte par votre assurance, car elle permet d'aider les parties à régler leur litige à l'amiable, hors des murs

\section{En plus du critère financier, qui joue un grand rôle, la qualité des prestations offertes est un élément central.}

4 Art. 35 CoD et 2 al. 1 du règlement du Bureau d'expertises extrajudiciaires de la FMH.

5 De telles assurances sont offertes par FMH Service société coopérative, leader sur le marché et partenaire de licence de la FMH. D'autres prestataires d'assurances sont la fmCh et Mediservice VSAOASMAC.

Correspondance: Valérie Rothhardt Avocate

Cheffe du Bureau d'exper-

tises extrajudiciaires

Fédération des médecins

suisses

Elfenstrasse 18

Postfach 300

CH-3000 Berne 15

valerie.rothhardt[at]fmh.ch d'un tribunal. En effet, l'expertise a pour but d'éclaircir la question de la faute de diagnostic ou de traitement; elle permet donc au patient d'obtenir des réponses à ses questions à des coûts raisonnables. Pour sa part, le médecin peut se voir éviter un éventuel procès en responsabilité civile et ainsi économiser du temps, de l'argent et de l'énergie.

Le Bureau d'expertises est également important pour la réputation du corps médical, car il permet de démontrer que ce dernier est soucieux de faire examiner des situations jugées critiques par le patient et de reconnaître ses fautes, dans le but d'améliorer la qualité de ses prestations et d'accroître la sécurité des patients.

Les membres de la FMH ont d'ailleurs l'obligation de se soumettre à une procédure devant le Bureau d'expertises extrajudiciaires de la FMH requise par le patient et acceptée par le bureau d'expertises. ${ }^{4}$ En 1982, l'ASA (Association Suisse d'Assurances, anciennement ARCA) et la FMH ont conclu un gentlemen's agreement qui stipule que les honoraires des experts pour des procédures devant le Bureau d'expertises extrajudiciaires de la FMH seront pris en charge par les assurances RC. Cette prise en charge ne dépend pas du résultat de l'expertise. Ce gentlemen's agreement, toujours en vigueur aujourd'hui, est automatiquement applicable aux assurances membres de l'ASA. Par contre, lorsque l'assurance RC n'est pas membre de l'ASA, la prise en charge des honoraires des experts n'est pas garantie. Le médecin peut accepter de prendre lui-même en charge les honoraires, mais n'y est pas contraint. Sans garantie de paiement des honoraires, l'expertise ne peut pas être organisée; le patient est donc privé d'une possibilité de faire éclaircir la question de la faute professionnelle de manière extrajudiciaire et peut être ainsi poussé à s'adresser plus facilement à un tribunal pour faire valoir ses prétentions.

\section{Recommandation}

En tant que médecin indépendant, vous êtes responsable du fait d'avoir une couverture d'assurance RC adéquate et adaptée à vos besoins.

Pour s'assurer de la qualité des prestations fournies, la FMH recommande à ses membres de vérifier que leur assurance RC soit membre de l'ASA, respectivement de

Pour s'assurer de la qualité des prestations fournies, la FMH recommande de conclure une assurance RC uniquement avec une assurance membre de l'ASA.

conclure une assurance RC uniquement avec une assurance membre de l'ASA.

Demandez conseil à des professionnels en la matière, neutres et compétents 5 . 